\title{
Rate Maximization in Multi-Antenna Broadcast Channels with Linear Preprocessing
}

\author{
Mihailo Stojnic, Haris Vikalo and Babak Hassibi \\ Department of Electrical Engineering \\ California Institute of Technology \\ Pasadena, California, USA \\ E-mail:\{mihailo, hvikalo, hassibi\}@systems.caltech.edu
}

\begin{abstract}
The sum rate capacity of the multi-antenna broadcast channel has recently been computed. However, the search for efficient practical schemes that achieve it is still ongoing. In this paper, we focus on schemes with linear preprocessing of the transmitted data. We propose two criteria for precoding matrix design, one maximizing the sum rate and the other maximizing the minimum rate among all users. The latter problem is shown to be quasiconvex and is solved exactly via bisection method. In addition to precoding, we employ signal scaling scheme that minimizes average bit-error-rate (BER). The signal scaling scheme is posed as a convex optimization problem, and thus can be solved exactly via efficient interior-point methods. In terms of achievable sum rate, the proposed technique significantly outperforms traditional channel inversion methods, while having comparable (in fact, often superior) BER performance.
\end{abstract}

\section{INTRODUCTION}

Recently, achievable limits of the performance of a multiantenna broadcast channel have been intensively studied (see, e.g., [1], [2], and the references therein). In [3], [4], non-linear techniques that attempt to approach those limits have been considered. However, these schemes are often computationally prohibitive when the number of transmit antennas is large. In this paper, we limit ourselves to linear data preprocessing at the transmitter and, under such a constraint, find a precoding scheme maximizing the sum-rate of the broadcast channel. We also consider linear precoding schemes that maximize the minimum rate among the users. The latter problem is shown to be quasiconvex and is solved exactly using efficient interior point methods. In addition to the precoding, we minimize the average BER among the users by performing an appropriate signal scaling. The best performance is obtained when the optimal preprocessing and signal scaling are combined.

We assume a standard system model for the broadcast channel with $M$ transmit antennas and $M$ users, described by

$$
\mathbf{r}=H \mathbf{s}+\mathbf{w}
$$

where $H$ is an $M \times M$ fading channel matrix whose entries are i.i.d. zero-mean, unit variance, complex Gaussian random variables, and $\mathbf{w}$ is an $M \times 1$ vector whose entries are also i.i.d. zero-mean, variance $\sigma^{2}$ complex Gaussian random variables

This work was supported in part by the National Science Foundation under grant no. CCR-0133818, by the Office of Naval Research under grant no. N00014-02-1-0578, and by Caltech's Lee Center for Advanced Networking which represent additive noise at each receiver. Furthermore, $\mathrm{s}$ is an $M \times 1$ vector of signals sent from the transmit antennas, and $\mathbf{r}$ is an $M \times 1$ vector whose components are the received signals at each user. The transmitted vector $\mathbf{s}$ is assumed to be obtained by linear preprocessing of the information vector $\mathbf{u}$, $\mathbf{s}=k G \mathbf{u}$, where $\mathbf{u}=\left[u_{1}, u_{2}, \ldots, u_{M}\right]^{T}$ and $u_{1}$ is the symbol intended for the first user, $u_{2}$ is intended for the second user and so on, and where $k$ is a scaling coefficient which ensures that the power constraint is satisfied.

We proceed by first designing $G$ to either maximize the sum rate or to maximize the minimum rate among all users. Then we show how to optimize over vectors $\mathbf{u}$ in order to minimize average BER in the system.

\section{Finding OPTIMAL PREPROCESSING MATRIX $G$}

In this section, we find the optimal preprocessing matrix $G$ assuming average transmit power constraint, $E\|\mathbf{s}\|^{2}=1$. Without a loss of generality, we will assume that $E \mathbf{u u}^{*}=I$. Then $E\|G \mathbf{u}\|^{2}=E \operatorname{tr}\left(G^{*} G \mathbf{u u}^{*}\right)=\operatorname{tr}\left(G^{*} G\right)$ and thus $k=$ $1 / \sqrt{\operatorname{tr}\left(G^{*} G\right)}$. Hence, from (1) we obtain

$$
\mathbf{r}=\frac{H G \mathbf{u}}{\sqrt{\operatorname{tr}\left(G^{*} G\right)}}+\mathbf{w}
$$

The matrix $G$ in (2) should be designed to optimize the performance of the overall system in terms of both the rate as well as the bit error rate. Often encountered in literature is the solution employing an regularized-pseudo inverse of the channel matrix $H G=H^{*}\left(\beta I+H H^{*}\right)^{-1}$, where the coefficient $\beta$ is typically chosen to maximize the signal-to-interference and noise ratio (SINR) (see, e.g., [4]). However, optimizing for SINR does not necessarily imply that the total sum rate will be maximized. This justifies the search for a better choice for the matrix $G$.

We consider two optimization criteria for the design of the preprocessing matrix $G$. First, we maximize the total sum rate over the space of all $M \times M$ complex matrices $G$. As we shall see, this optimization results in a strategy where at each channel use, a subset of users is chosen and data transmitted only to those users. Second, we consider the problem of optimal preprocessing that maximizes the minimum rate among all the users. Extensive simulations imply that the best BER performance of the system is achieved when the two strategies are 
combined, i.e., when a subset of users is selected and then the minimum rate among the users in that subset is maximized.

\section{A. Maximizing the sum rate over $G$}

We assume that each user treats the interference as noise. Therefore the sum rate of the broadcast channel (2) is given by $R=\sum_{m=1}^{M} \log \left(1+\frac{\left|\sum_{p} H_{m p} G_{p m}\right|^{2}}{\sigma^{2} \operatorname{tr}\left(G^{*} G\right)+\sum_{n \neq m}\left|\sum_{p} H_{m p} G_{p n}\right|^{2}}\right)$. The optimal choice for the matrix $G$ is the solution to the optimization problem

$$
\max _{G} R
$$

A closed-form analytic solution to (3) does not appear easy to find and thus we solve it iteratively. Before proceeding any further, we will find it useful to define $\operatorname{num}_{m}=\left|\sum_{p=1}^{M} H_{m p} G_{p m}\right|^{2}, \quad \operatorname{den}_{m}=\sigma^{2} \operatorname{tr}\left(G^{*} G\right)+$ $\sum_{n=1, n \neq m}^{M}\left|\sum_{p=1}^{M} H_{m p} G_{p n}\right|^{2}$ The following lemma gives a necessary condition for the optimal $G$.

Lemma 1: Any $G$ which is the solution of (3), is of the form $G=\left(\left(\sigma^{2} \operatorname{tr} D\right) I+H^{*} D H\right)^{-1} H^{*} \Delta$, where $\Delta=\operatorname{diag}\left(\frac{(H G)_{11}}{\operatorname{den}_{1}}, \ldots, \frac{(H G)_{l l}}{\operatorname{den}_{l}}, \ldots, \frac{(H G)_{M M}}{\operatorname{den}_{M}}\right)$ and $D=$ $\operatorname{diag}\left(\frac{\operatorname{num}_{1}}{\operatorname{den}_{1}\left(\operatorname{den}_{1}+\operatorname{num}_{1}\right)}, \ldots, \frac{\operatorname{den}_{l}}{\left.\operatorname{nen}_{l} \operatorname{den}_{l}+\operatorname{num}_{l}\right)}, \ldots\right.$, $\left.\frac{\operatorname{nem}_{M}}{\operatorname{den}_{M}\left(\operatorname{den}_{M}+\operatorname{num}_{M}\right)}\right)$

Proof: It is sufficient to show that $\frac{\partial R}{\partial G_{k l}}=0 \Rightarrow$ $G=\left(\left(\sigma^{2} \operatorname{tr} D\right) I+H^{*} D H\right)^{-1} H^{*} \Delta$. It is straightforward to show that $\frac{\partial R}{\partial G_{k l}}=\frac{H_{l k}(H G)_{l l}^{*}}{\operatorname{den}_{l}}-\sum_{m=1}^{M} \frac{\operatorname{num}_{m} H_{m k}(H G)_{m l}^{*}}{\operatorname{den}_{m}\left(\operatorname{num}_{m}+\operatorname{den}_{m}\right)}-$ $\sum_{m=1}^{M} \frac{\sigma^{2} G_{k l}^{*} \text { num }_{m}}{\operatorname{den}_{m}\left(\operatorname{den}_{m}+\text { num }_{m}\right)}$. Setting each of these derivatives to zero we obtain $H^{*} \Delta-H^{*} D H G-\left(\sigma^{2} \operatorname{tr} D\right) G=0$, or equivalently $G=\left(\left(\sigma^{2} \operatorname{tr} D\right) I+H^{*} D H\right)^{-1} H^{*} \Delta$. This implies that $\frac{\partial R}{\partial G_{\text {hl }}}=0 \Rightarrow G=\left(\left(\sigma^{2} \operatorname{tr} D\right) I+H^{*} D H\right)^{-1} H^{*} \Delta$, which concludes the proof.

Using Lemma 1, we state the following iterative algorithm for solving (3).

$$
D_{0}=I, \Delta_{0}=I, i=0
$$

\section{Repeat}

1) $G_{i}=\left(\left(\sigma^{2} \operatorname{tr} D_{i}\right) I+H^{*} D_{i} H\right)^{-1} H^{*} \Delta_{i}$

2) num $_{m}=\left|\sum_{p=1}^{M}\left(H G_{i}\right)_{m m}\right|^{2}$

3) $\operatorname{den}_{m}=\sigma^{2} \operatorname{tr}\left(G_{i}^{*} G_{i}\right)+\sum_{n=1, n \neq m}^{M}\left|\sum_{p=1}^{M}\left(H G_{i}\right)_{m n}\right|^{2}$

4) $D_{i+1}=\operatorname{diag}\left(\frac{\text { num }_{1}}{\operatorname{den}_{1}\left(\text { den }_{1}+\text { num }_{1}\right)}, \ldots, \frac{\text { num }_{l}}{\operatorname{den}_{l}\left(\operatorname{den}_{l}+\text { num }_{l}\right)}\right.$, $\left.\ldots, \frac{\operatorname{num}_{M}}{\operatorname{den}_{M}\left(\operatorname{den}_{M}+\text { num }_{M}\right)}\right)$,

5) $\Delta_{i+1}=\operatorname{diag}\left(\frac{\left(H G_{i}\right)_{11}}{\operatorname{den}_{1}}, . ., \frac{\left(H G_{i}\right)_{l l}}{\operatorname{den}_{l}}, \ldots, \frac{\left(H G_{i}\right)_{M M}}{\operatorname{den}_{M}}\right)$

6) $i=i+1$

end

We refer to using the matrix $G$ obtained from the previous iterative procedure as Method 2.1. Since $H^{*}\left(\left(\sigma^{2} \operatorname{tr} D\right) I+\right.$ $\left.H H^{*}\right)^{-1}=\left(\left(\sigma^{2} \operatorname{tr} D\right) I+H^{*} H\right)^{-1} H^{*}$, the initial value $G_{0}$ coincides with the one obtained by the regularized pseudo-inverse (see, e.g., [4]). Simulation results presented in following sections imply that such a choice of initial value leads to an iterative process that converges after a fairly small number of iterations (roughly 15 on average). As illustrated by Figure 1 and Figure 2, the matrix $G$ obtained in this manner significantly outperforms regularized inverse solution.

\section{B. Maximizing the minimum rate over $G$}

Instead of maximizing sum rate, one may demand that even the worst (active) user gets as large rate as possible. This criterion leads to the following optimization problem

$$
\max _{G} \min _{i} \log \left(1+\frac{\left|(H G)_{i i}\right|^{2}}{\sigma^{2} \operatorname{tr}\left(G^{*} G\right)+\sum_{j, j \neq i}\left|(H G)_{i j}\right|^{2}}\right) .
$$

The previous problem (or problems similar to it) have been studied and various algorithms for solving it have been suggested throughout the literature (see, e.g. [5], [6], [7], [8]). Here we suggest another way of solving it based on iterior-point methods. Define $B=H G$. Then (4) can be written as

$$
\max _{B} \min _{i} \frac{\left|B_{i i}\right|^{2}}{\sigma^{2} \operatorname{tr}\left(B^{*} H^{-*} H^{-1} B\right)+\sum_{j, j \neq i}\left|B_{i j}\right|^{2}} .
$$

Without a loss of generality, we can assume that the optimal $B_{i i}$ are real and positive. [Because multiplying $B$ by a diagonal unitary phase matrix does not change the value of objective function in (5).] Let $\operatorname{vec}(B)$ denote a vector comprised of columns of matrix $B$, i.e., the first $M$ elements of $\operatorname{vec}(B)$ are the first column of $B$, the second $M$ elements of $\operatorname{vec}(B)$ are the second column of $B$ and so on. Then we can write $\sigma^{2} \operatorname{tr}\left(B^{*} H^{-*} H^{-1} B\right)=\sigma^{2} \operatorname{vec}(B)^{*}\left(I \otimes H^{-*} H^{-1}\right) \operatorname{vec}(B)$. Defining $F=I \otimes H^{-*} H^{-1}$, we further have

$$
\begin{aligned}
& \sigma^{2} \operatorname{tr}\left(B^{*} H^{-*} H^{-1} B\right)=\sigma^{2} \operatorname{vec}(B)^{*} F \operatorname{vec}(B) \\
& \quad=\sigma^{2}\left[\begin{array}{l}
\Re(\operatorname{vec}(B)) \\
\Im(\operatorname{vec}(B))
\end{array}\right]^{*}\left[\begin{array}{cc}
\Re(F) & -\Im(F) \\
\Im(F) & \Re(F)
\end{array}\right]\left[\begin{array}{l}
\Re(\operatorname{vec}(B)) \\
\Im(\operatorname{vec}(B))
\end{array}\right]
\end{aligned}
$$

Let $\mathbf{x}=\left[\begin{array}{c}\Re(\operatorname{vec}(B)) \\ \Im(\operatorname{vec}(B))\end{array}\right]$ and $T=\left[\begin{array}{cc}\Re(F) & -\Im(F) \\ \Im(F) & \Re(F)\end{array}\right]$. Then we can write

$$
\sigma^{2} \operatorname{tr}\left(B^{*} H^{-*} H^{-1} B\right)=\sigma^{2} \mathbf{x}^{*} T \mathbf{x}
$$

Define $2 M^{2} \times 2 M^{2}$ matrix $K^{(i j)}$ with $K_{(j-1) M+i,(j-1) M+i}^{(i j)}=$ $K_{M^{2}+(j-1) M+i, M^{2}+(j-1) M+i}^{(i j)}=1$ and zeros otherwise. Using this $K^{(i j)}$ and (6), we can write (5) as

$$
\begin{array}{cl}
\min _{\mathbf{x}} \max _{i} & \frac{\mathbf{x}^{*} W_{i} \mathbf{x}}{x_{(i-1) M+i}^{2}} \\
\text { subject to } & x_{(i-1) M+i}>0, \quad 1 \leq i \leq M \\
& x_{M^{2}+(i-1) M+i}=0, \quad 1 \leq i \leq M,
\end{array}
$$

where $W_{i}=\sigma^{2} T+\sum_{j=1, j \neq i}^{M} K^{(i j)}$. Note that $W_{i}$ is positive semidefinite because matrices $T$ and $K^{(i j)}$ are positive semidefinite. To solve (7), we first consider the following lemma.

\section{Lemma 2: Optimization problem (7) is quasiconvex.}

Proof: We first need to prove that function $f_{i}(\mathbf{x})=$ $\frac{\mathbf{x}^{*} W_{i} \mathbf{x}}{x_{(i-1) M+i}^{2}}$ is quasiconvex. We can write $f_{i}(\mathbf{x})=\frac{g_{i}(\mathbf{x})}{x_{(i-1) M+i}}$, where $g_{i}(\mathbf{x})=\frac{\mathbf{x}^{*} W_{i} \mathbf{x}}{x_{(i-1) M+i}}$. Let us show that the function $g_{i}(\mathbf{x})$ is convex for $x_{(i-1) M+i}>0$. To do so, we need to show that

$$
g_{i}(\theta \mathbf{x}+\gamma \mathbf{y}) \leq \theta g_{i}(\mathbf{x})+\gamma g_{i}(\mathbf{y})
$$


where $g_{i}(\theta \mathbf{x}+\gamma \mathbf{y})=\frac{\theta^{2} \mathbf{x}^{*} W_{i} \mathbf{x}+2 \theta \gamma \mathbf{x}^{*} W_{i} \mathbf{y}+\gamma^{2} \mathbf{y}^{*} W_{i} \mathbf{y}}{\theta x_{(i-1) M+i}+\gamma y_{(i-1) M+i}}, g_{i}(\theta \mathbf{x})=$ $\theta \frac{\mathbf{x}^{*} W_{i} \mathbf{x}}{x_{(i-1) M+i}}, g_{i}(\gamma \mathbf{y})=\gamma \frac{\mathbf{y}^{*} W_{i} \mathbf{y}}{y_{(i-1) M+i}}$, and where $\theta+\gamma=1,0 \leq$ $\theta, \gamma \leq 1$. This is equivalent to showing that

$$
\frac{y_{(i-1) M+i}}{x_{(i-1) M+i}} \mathbf{x}^{*} W_{i} \mathbf{x}-2 \mathbf{x}^{*} W_{i} \mathbf{y}+\frac{x_{(i-1) M+i}}{y_{(i-1) M+i}} \mathbf{y}^{*} W_{i} \mathbf{y} \geq 0
$$

Since $W_{i}$ is symmetric and positive semidefinite, it can be written as $W_{i}=R_{i}^{*} R_{i}$. From Cauchy-Schwartz inequality it follows that

$$
\mathbf{x}^{*} W_{i} \mathbf{y}=\mathbf{x}^{*} R_{i}^{*} R_{i} \mathbf{y} \leq\left\|R_{i} \mathbf{x}\right\|_{2}\left\|R_{i} \mathbf{y}\right\|_{2}=\sqrt{\mathbf{x}^{*} W_{i} \mathbf{x y} \mathbf{y}^{*} W_{i} \mathbf{y}}
$$

Using (9), we obtain

$$
\begin{aligned}
& \frac{y_{(i-1) M+i}}{x_{(i-1) M+i}} \mathbf{x}^{*} W_{i} \mathbf{x}-2 \mathbf{x}^{*} W_{i} \mathbf{y}+\frac{x_{(i-1) M+i}}{y_{(i-1) M+i}} \mathbf{y}^{*} W_{i} \mathbf{y} \\
& \geq\left(\sqrt{\frac{y_{(i-1) M+i}}{x_{(i-1) M+i}} \mathbf{x}^{*} W_{i} \mathbf{x}}-\sqrt{\frac{x_{(i-1) M+i}}{y_{(i-1) M+i}} \mathbf{y}^{*} W_{i} \mathbf{y}}\right)^{2} \geq 0,
\end{aligned}
$$

hence proving (8). Therefore, function $g_{i}(\mathbf{x})$ is convex for $x_{(i-1) M+i}>0$. Using this fact, we now show that $f_{i}(\mathbf{x})$ is quasiconvex. Note that $\alpha$-sublevel sets of $f_{i}(\mathbf{x})$ are equivalent to the 0 - sublevel sets of the convex functions $g_{i}(\mathbf{x})-\alpha x_{(i-1) M+i}^{2}$. Since all sublevel sets of the convex function are convex, we conclude that any $\alpha$-sublevel set of function $f_{i}(\mathbf{x})$ is also convex. This is sufficient condition that function $f_{i}(\mathbf{x})$ is quasiconvex. To complete the proof of Lemma 2, recall that the pointwise maximum of the set of quasiconvex functions is quasiconvex. Therefore, the objective function in (7) is quasiconvex.

We use bisection method combined with interior-point method to solve (7). Once we found optimal $\mathbf{x}$ in (7), we determine $B$ such that $\mathbf{x}=\left[\begin{array}{l}\Re(\operatorname{vec}(B)) \\ \Im(\operatorname{vec}(B))\end{array}\right]$. Then we calculate $G$ as $G=H^{-1} B$. We refer to using the matrix $G$ found by the aforementioned procedure as Method 2.2.

The technique described in Subsection II-A maximizes the sum rate of the multi-antenna broadcast system under the linear data processing constraint. The individual rates resulting from the maximization (3), however, may differ significantly. This disparity is inherent to the optimization (3) since (3) essentially denotes the maximization of $\|\mathbf{v}\|_{1}$ (i.e., norm- 1 of the vector $\mathbf{v}$ ). It is well known that in the process of maximizing the norm1 of a vector, a few components of the vector are suppressed while the remaining ones are boosted up. Thus in Subsection II-A the sum rate is maximized at the expense of the weakest few users which are ignored. [Note: Transmitting data over many channel uses provides fairness.] The symbols intended for the remaining strong users may be modulated with higher modulation schemes, thus overcompensating for the sum rate seemingly lost by transmitting only to a subset of users.

On the other hand, as the result of the disparity among the individual rates (and hence among the SINRs and BERs of individual users), the average BER of the system may suffer. To compensate for the loss in average BER, we employ Method 2.2 on the subset of strong users selected for transmission by Method 2.1. As it turns out, maximizing the minimum individual rate among the selected strong users results in fairly equal (and high) SINRs. We refer to the combination of the procedures suggested in the previous two subsections as Method 2 and give its performances on Figures 1 and 2 . Although Method 2 selects only a subset of users, it provides much higher sum-rate per channel use than the scheme employing regularized pseudo-inverse. This happens because Method 2 allows the selected users to employ higher modulation schemes. The rate comparison is illustrated in Figure 2. The average BER performance of the two schemes (regularized pseudo-inverse with a low rate and Method 2 with significantly higher rate) are comparable (in fact, Method 2 often outperforms regularized pseudo-inverse), and are illustrated in Figure 1 .

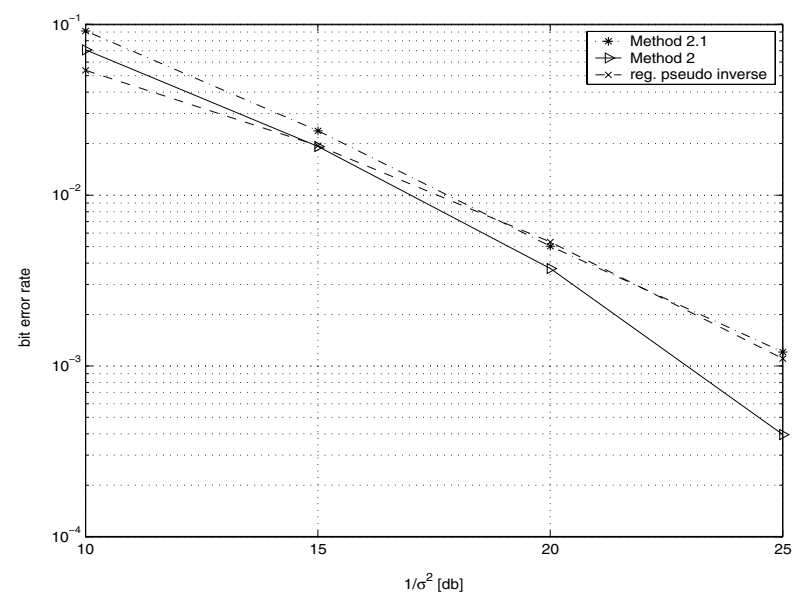

Fig. 1. Comparison of BER, $M=6$ antennas/users, 8PSK-Method 2, 8PSKMethod 2.1, 4PSK-regularized pseudo inverse

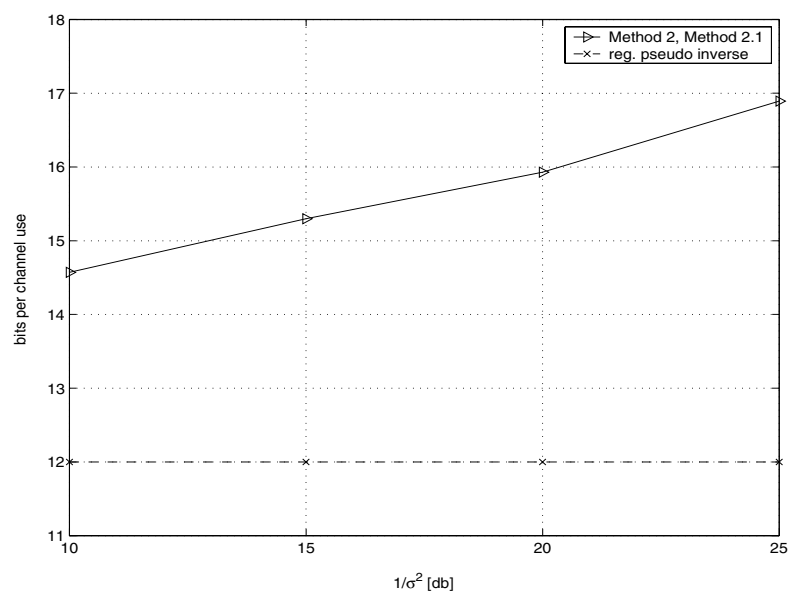

Fig. 2. Comparison of rates, $M=6$ antennas/users, 8PSK-Method 2, 8PSKMethod 2.1, 4PSK-regularized pseudo inverse

\section{FINDING OPTIMAL SIGNALING SCHEME}

Recall our basic model (1). Let assume that the preprocessing matrix $G$ is obtained by simple inversion of the channel matrix $H$, i.e., $G=H^{-1}$. In this section, we propose a way of scaling the magnitudes of the information signal $\mathbf{u}$ so as to minimize the average BER. [Note that in the next section we will 
show how to employ this signal scaling scheme to the optimal $G$ obtained in Section II.]

To minimize the average BER, one needs to maximize the minimum SINR at receivers. To this end, in [4] authors suggest perturbation of information signals by appropriately translating original $M$-QAM signal constellation in complex space. In this section we suggest a similar idea but focus on perturbations (in fact, radial scaling) of $M$-PSK constellation. An advantage of constraining ourselves to PSK constellations is in simplicity of decoding. Since the signal points are perturbed only radially, rather than vertically or horizontally as in QAM, the angular information has not changed. Therefore, no side information about the signaling scheme (i.e., the nature of the perturbation) is needed at the receiver. In other words, each user's decoder makes simple angular decisions. The decoder is no longer necessarily ML but it is efficient and practical since it requires no additional information from the transmitter. [Our simulation results indicate that the performance of this sub-optimal ML decoder is almost identical to the optimal one.]

In the following two subsections we are proposing two possible methods for this perturbation. [We should also note that the signal scaling scheme, presented below, will differ for general $G \neq H^{-1}$, as we will show in later section.]

\section{A. Finding optimal scaling coefficient $k$}

By fixing $G=H^{-1}$ and representing $\mathbf{u}$ via its phases and magnitudes, we can rewrite (1) as $\mathbf{r}=k H H^{-1} \Phi \mathbf{u}_{m}+\mathbf{w}$, where $\mathbf{u}=\Phi \mathbf{u}_{m}$ and $\Phi$ is the diagonal matrix of phases of $\mathbf{u}$, and where $\mathbf{u}_{m}$ is the vector of magnitudes of $\mathbf{u}$. Note that due to the use of a PSK modulation scheme, useful information is contained in $\Phi$. We are concerned with designing optimal magnitudes of the signals, i.e., designing $\mathbf{u}_{m}$. The relevant power constraint now becomes the one on instantaneous, rather than average, transmission power. This means that the corresponding form to (2) can be written as

$$
\mathbf{r}=\frac{H H^{-1} \Phi \mathbf{u}_{m}}{\sqrt{\operatorname{tr}\left(\mathbf{u}_{m}^{*} \Phi^{*} H^{-*} H^{-1} \Phi \mathbf{u}_{m}\right)}}+\mathbf{w} .
$$

Now we want to optimize scaling coefficient while keeping magnitudes of $\mathbf{u}$ greater or equal to 1 . This will result in magnitudes of the components of the received vector $\mathbf{r}$ that are at least as large as if there were no signal scaling at all. This requires solving the following optimization problem

$$
\begin{aligned}
\min & \mathbf{u}_{m}^{*} \Phi^{*} H^{-*} H^{-1} \Phi \mathbf{u}_{m} \\
\text { subject to } & u_{m_{i}} \geq 1, \quad 1 \leq i \leq M
\end{aligned}
$$

This problem is convex and can easily be solved exactly by a host of numerical methods (see, e.g., [9] and the references therein). More importantly, we can show that the solution of this problem is equal to the solution to

$$
\begin{aligned}
\max _{u_{m_{1}}, u_{m_{2}}, \ldots, u_{m_{M}}} \min _{i} & \frac{u_{m_{i}}^{2}}{\mathbf{u}_{m}^{*} \Phi^{*} H^{-*} H^{-1} \Phi \mathbf{u}_{m}} \\
\text { subject to } & u_{m_{i}} \geq 1, \quad 1 \leq i \leq M,
\end{aligned}
$$

which is the problem of maximizing the minimum SINR in system (10). Denoting by $\widehat{\mathbf{u}_{m}}$ the solution to (12), we see that the transmitted signal should have the form of $\mathbf{s}=$ $\frac{H^{-1} \Phi \widehat{\mathbf{u}_{m}}}{\sqrt{\widehat{\mathbf{u}_{m}} \Phi^{*} H^{-*} H^{-1} \Phi \widehat{\mathbf{u}_{m}}}}$. We refer to this signal scaling policy as Method 3.1. As said earlier, although the magnitudes of optimal $\mathbf{u}$ will generally be different from 1 , the receivers will still be able to decode received signals by considering their angle, since $\mathbf{s}$ has the same phase matrix $\Phi$ as $\mathbf{u}$.

\section{B. Maximizing the virtual rate over $\mathbf{u}$}

The rate function defined at the beginning of Subsection II-A is not applicable anymore, since we are performing optimization over the transmitted signal. However, motivated by the Methods suggested in Section II, we propose an optimization of a similar objective function, which results in further improvement of the BER. Recall that the Method 2.1 essentially suppresses transmission to the set of users that have particularly bad SINR. Then the Method 2.2 maximized the minimum SINR among the remaining users. Here we propose the same technique, only by focusing on what we will refer to as virtual rate function $R_{v}, R_{v}=\sum_{i=1}^{M} \log \left(1+\frac{u_{m_{i}}^{2}}{\mathbf{u}_{m}^{*} \Phi^{*} H^{-*} H^{-1} \Phi \mathbf{u}_{m}}\right)$. Numerical maximization of $R_{v}$ over $u_{m_{i}}$ is fairly straightforward. As a solution we obtain the set $\widehat{u_{m_{i}}}, 1 \leq i \leq M$. Following the methodology of Method 2, those $u_{m_{i}}$ 's that are below some threshold are set to 0; then (12) is solved over the other components of $\mathbf{u}_{m}$. As the result of this procedure, we obtain higher SINRs $\frac{u_{m_{i}}^{2}}{\mathbf{u}_{m}^{*} \Phi^{*} H^{-*} H^{-1} \Phi \mathbf{u}_{m}}$ for the set of active users than what we would have obtained by applying only Method 3.1. This, in turn, gives us an option to operate with higher constellation and increase the sum rate while preserving the average BER. We refer to aforementioned modification of Method 3.1 as Method 3. The performances of Method 3.1 and Method 3 are given in Figure 3 and Figure 4. As evident from these figures, both algorithms significantly outperform regularized inverse, both in terms of BER and sum rate. Compared to performance of the system with regularized inverse, Method 3.1 significantly improves BER at high SNR, while Method 3 operates on higher rates while preserving roughly the same BER.

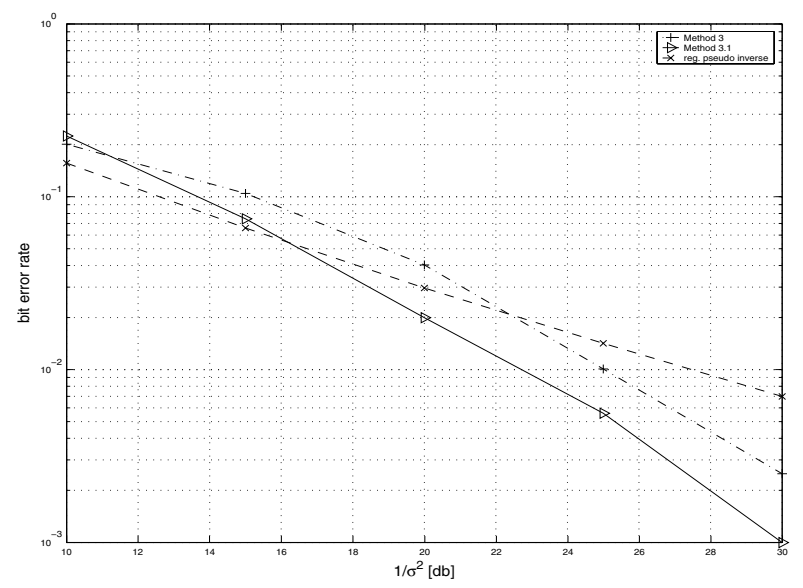

Fig. 3. Comparison of BER, $M=20$ antennas/users, 16PSK-Method 3, 8PSKMethod 3.1, 8PSK-regularized pseudo inverse 


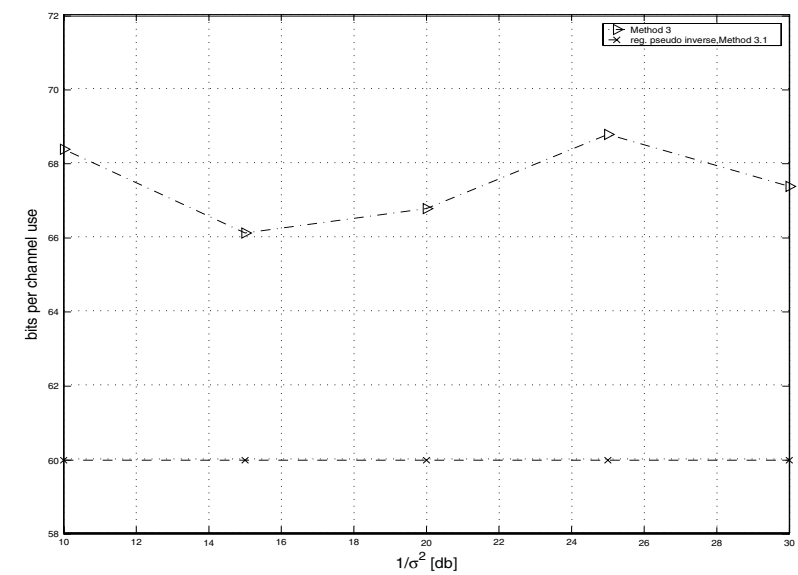

Fig. 4. Comparison of rates, $M=20$ antennas/users, 16PSK-Method 3, 8PSKMethod 3.1, 8PSK-regularized pseudo inverse

\section{COMBined Method}

In Section III, we employed the signal scaling scheme to optimize the BER in a system that uses $G=H^{-1}$ for data preprocessing. In this section, we combine the signal scaling with the optimal preprocessing matrices $G$ found in Section II. This is done in stages. In particular, assume that Method 2.1 is used to find $G$ which maximizes the sum rate of the channel. Then to minimize average BER of the users, we employ signal scaling for such $G$. Instead of solving (11) (which assumed $G=H^{-1}$ ), we now need to solve optimization

$$
\begin{aligned}
\min & \mathbf{u}_{m}^{*} \Phi^{*} \widehat{G}^{*} \widehat{G} \Phi \mathbf{u}_{m} \\
\text { subject to } & u_{m_{i}} \geq 1, \quad 1 \leq i \leq M,
\end{aligned}
$$

where $\widehat{G}$ is $G$ found by Method 2.1. The above problem is convex and thus can be solved exactly via efficient convex optimization techniques. If we denote solution of (13) by $\widehat{\mathbf{u}_{m}}$, the optimal transmitted signal $\mathbf{s}$ is given by $\mathbf{s}=\frac{\widehat{G} \Phi \widehat{\mathbf{u}_{m}}}{\sqrt{\widehat{\mathbf{u}_{m}}{ }^{*} \widehat{G}^{*} \widehat{G} \Phi \widehat{\mathbf{u}_{m}}}}$. We refer to the above algorithm as Method 4 and give its performances on the Figures 5 and 6. It can be seen that Method 4 outperforms Method 2 in terms of BER. Both methods provide the same sum rate significantly higher than the sum rate of regularized pseudo-inverse.

\section{CONClusion}

In this paper, we have proposed two criteria for the design of precoding matrix in a multi-antenna broadcast system. First, we maximized the sum rate, and then we showed how to maximize the minimum rate among all users. The latter problem is shown to be quasiconvex and solved exactly. The precoding techniques are constrained to linear preprocessing at the transmitter. In addition to precoding, we have employed signal scaling scheme that minimizes the average BER of the users. The signal scaling scheme is posed as a convex optimization problem, and solved exactly via interior-point methods. Finally, we have combined the precoding with signal scaling. The combined scheme can be efficiently applied in practice. In terms of achievable sum rate, the proposed technique significantly outperforms traditional channel inversion methods, while having comparable (in fact, often superior) BER performance.

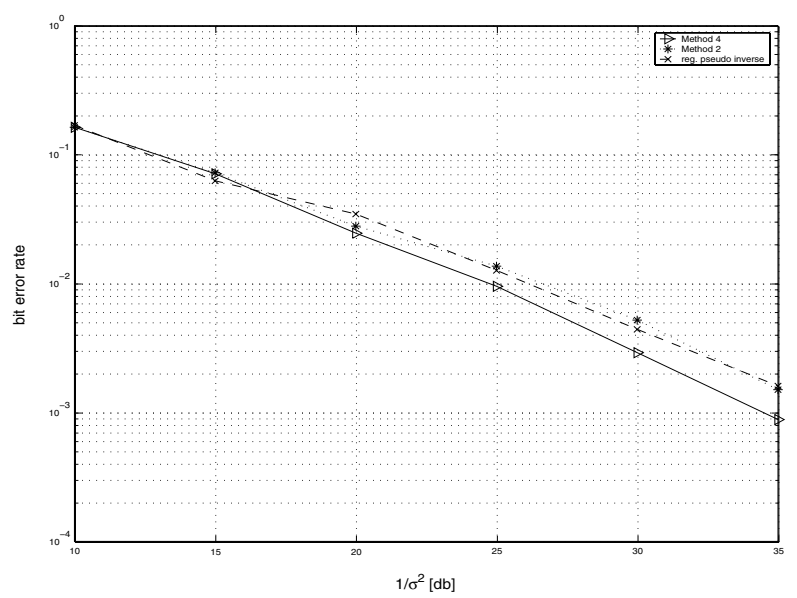

Fig. 5. Comparison of BER, $M=6$ antennas/users, 16PSK-Method 4, 16PSKMethod 2,8PSK-regularized pseudo inverse

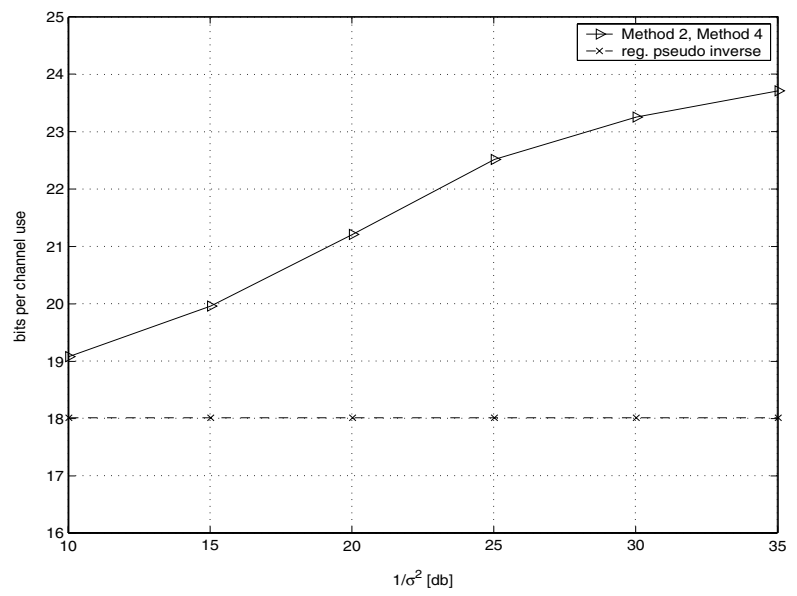

Fig. 6. Comparison of rates, $M=6$ antennas/users, 16PSK-Method 4, 16PSKMethod 2,8PSK-regularized pseudo inverse

\section{REFERENCES}

[1] G.Caire and S.Shamai, "On the achievable throughput of a multiantenna gaussian broadcast channel," IEEE Trans. on Inf. Theory, July 2003.

[2] S.Wishwanath, N.Jindal, and A.Goldsmith, "Achievable rates, and sumrate capacity of gaussian mimo broadcast channels," IEEE Trans. on Inf. Theory, Oct. 2003.

[3] R.Zamir, S.Shamai, and U.Erez, "Nested linear/lattice codes for structured multiterminal binning," IEEE Trans. on Inf. Theory, June 2002.

[4] C.Peel, B.Hochwald, and A. Swindlehurst, "A vector-perturbation technique for near-capacity multi-antenna multi-user," submitted to IEEE Trans. of Communications, June 2003.

[5] J.-H. Chang, L. Tassiulas, and F. Rashid-Farrokhi, "Joint transmitter receiver diversity for efficient space division multiaccess," IEEE Trans. on Wireless Communications, Jan. 2002.

[6] J. Bertrand and P. Forster, "Optimal weights computation of an emitting antenna array-the obele algorithm," IEEE Trans. on Signal Processing, July 2003.

[7] E. Visotsky and U. Madhow, "Optimum beamforming using transmit antenna arrays," IEEE Vehicular Technology Conference, May 1999.

[8] A.Wiesel, Y.C.Eldar, and S.Shamai, "Multiusers precoders for fixed receivers," International Zurich Seminar on Communications, 2004.

[9] S.Boyd and L.Vandenberghe, Convex Optimization, Cambridge University Press, 2003. 